Review

\title{
Molecular Mechanisms and Countermeasures of Immunotherapy Resistance in Malignant Tumor
}

\author{
Xiaoyue Jiang ${ }^{1}, \mathrm{Li} \mathrm{Li}^{1}$, Yingrui $\mathrm{Li}^{1,2}$, Qin $\mathrm{Li}^{1}{ }^{\bowtie}$ \\ 1. Department of Oncology, Beijing Friendship Hospital, Capital Medical University, Beijing, 100050, China. \\ 2. Department of Biochemistry \& Molecular Biology, School of Basic Medical Sciences, Shanxi Medical University, Taiyuan,030000, China. \\ $\square$ Corresponding author: Dr. Qin Li. Department of Oncology, Beijing Friendship Hospital, Capital Medical University, Beijing, 100050, China. Tel.: +86 10 \\ 63139325; Fax: +86 10 63139325. E-mail: oncologistinbj@163.com. \\ (C) Ivyspring International Publisher. This is an open access article distributed under the terms of the Creative Commons Attribution (CC BY-NC) license \\ (https://creativecommons.org/licenses/by-nc/4.0/). See http://ivyspring.com/terms for full terms and conditions
}

Received: 2018.04.04; Accepted: 2019.02.21; Published: 2019.04.20

\begin{abstract}
Tumor immunotherapy inhibits the proliferation and invasion of tumor cells by inducing or enhancing anti-tumor immune responses in active or passive ways. It is the fourth therapeutic method with efficiency and safety in addition to surgery, radiotherapy and chemotherapy. At present, anti-tumor immune related clinical trials have made promising achievements in prolonging progression free survival and overall survival, therefore, FDA approved a variety of immune checkpoint blockers (ICBs) such as nivolumab, pembrolizumab, ipilimumab. However, primary or acquired resistance results in massive perplexity to oncologist and patients. In order to bring further clinical benefit to tumor patients, study on mechanisms of immunotherapy resistance is extremely urgent. This review summarizes related mechanisms of tumor immunotherapy resistance, including MITF suppression, Ezh2 upregulation, TIM-3 upregulation, microRNA-driven deregulation of cytokine expression and et al. Genetic mutations such as PTEN loss, JAK1/2 loss-of-function mutations and $\mathrm{Cbl}-\mathrm{b}$ deficiency are also involved. Moreover, we have discussed feasible countermeasures, for instance, combining ICBs with PRRs agonists, ARNAX, CPG oligonucleotide, oncolytic peptide LTX-315 and indoleamine 2, 3-dioxygenase inhibitors, respectively. Other methods include combined ICBs with radiotherapy, combined ICBs with blockade of PI3K-AKT, TIM-3 pathway; blockade of Fcy receptors before anti-PD-1 monoclonal antibodies administration and modulation of the gut microbiome, et al. Mechanisms and countermeasures of immunotherapy resistance still requires further exploration, in expectation to provide novel ideals and basis for tolerant patients.
\end{abstract}

Key words: immunotherapy, resistance, checkpoint, combination therapy

\section{Introduction}

Tumor immunotherapy inhibits the proliferation and invasion of tumor cells by inducing or enhancing anti-tumor immune responses in active or passive ways. It is the fourth therapeutic method with efficiency and safety in addition to surgery, radiotherapy and chemotherapy. It is mainly divided into passive immunotherapy and active immunotherapy. Passive immunotherapy includes monoclonal antibody $(\mathrm{mAb})$ and antibody-drug conjugate while active immunotherapy contains cancer vaccine and chimeric antigen receptor $\mathrm{T}$-cell immunotherapy $(\text { CAR-T })^{1}$.
Currently, many clinical trials related to immunotherapy have made remarkable achievements. Phase II - IV clinical trials show that immune checkpoint blockers (ICBs) significantly prolong progression free survival, overall survival in melanoma ${ }^{2,3,4}$ non-small cell lung cancer (NSCLC) $)^{5,6}$ head and neck squamous cell carcinoma (HNSCC $)^{7}$, liver cancer ${ }^{8}$, colorectal cancer ${ }^{9}$, urothelium carcinoma ${ }^{10}$, renal cell carcinoma ${ }^{11}$, classical Hodgkin lymphoma ${ }^{12}$ and gastric cancer ${ }^{13}$. Hence, FDA has approved a variety of ICBs for clinical therapy in multiple tumors. Ipilimumab has been approved for 
melanoma, while nivolumab and pembrolizumab have been approved for not only melanoma but also other malignant tumors. Meta-analysis showed that the validity and safety of PD-1/PD-L1 inhibitors are prior in NSCLC patients when compared to docetaxel-based chemotherapy, especially in prolonging progression free survival and overall survival ${ }^{14}$.

\section{Resistance occurrence in clinical immunotherapy}

Clinicians and patients are high with the hope of curing cancer, for the immunotherapy superiority. However, some patients show innate resistance while other patients develop acquired resistance after several courses of immunotherapy. Schadendorf, et al summarized several clinical trials (ID\# CA184-00715, CA184-00816 and CA184-02217) and demonstrated that $25 \%$ of 88 metastatic melanoma patients had primary resistance $^{18}$. According to pooled analysis in 655 advanced metastatic melanoma patients, the twelvemonth progression-free survival rate was $35 \%$ and the median overall survival was 23 months, yet $25 \%$ patients appeared recurrence and resistance at 21 months ${ }^{19}$. Ipilimumab, an anti-CTLA-4 mAb, has better effect on survival improvement in BRAF V600E-negative malignant melanoma compared to radiotherapy and chemotherapy. Despite the significant prolonged progression-free survival and overall survival, the majority of patients developed resistance within one year ${ }^{20}$. Hence, primary and acquired resistance results in massive perplexity to oncologist and patients, making study on mechanisms of immunotherapy resistance extremely urgent in order to bring better clinical benefit to tumor patients.

Optimal management of immunotherapy is needed to avoid resistance. There are two major challenges in management optimization: identification of sensitive patients and exploration of immunotherapy resistance mechanism. Gene mutation detection partly identifies primary and acquired resistance ${ }^{21,22}$. Resistance to ICBs differentiates in patients and tumors. Analyzing mechanisms of tumor immunologic escape in different levels conduces to formulating corresponding countermeasures ${ }^{23}$. This review summarizes related mechanisms of immunotherapy resistance and feasible countermeasures in expectation to improve prognosis of immunotherapy resistant patients.

\section{Occurrence of resistance to immunotherapy}

Research on anti-PD-1 therapy showed that key genetic mutations, antigen presentation alternations and loss of the IFN- $\gamma$ signaling pathway related genes can effectively block the ongoing therapeutic immune response ${ }^{24}$. Immunogenicity diversity caused by genetic mutations is the major determinant of immunotherapy resistance. The efficacy of immunotherapy is determined by gene expression profile differential ${ }^{25}$. Primary or acquired resistance to tumor immunotherapy arises in multiple ways, including microphthalmia-associated transcription factor (MITF) suppression, increased enhancer of zeste homolog 2 (Ezh2) expression, microRNA-driven deregulation of cytokine expression, $\mathrm{T}$ cell immunoglobulin mucin-3 (TIM-3) upregulation and Wnt/ $\beta$-catenin signaling pathway activation. Genetic mutations such as phosphatase and tensin homolog deleted on chromosome ten (PTEN) loss, Janus Kinase 1/2 (JAK1/2) loss-of-function mutations and Casitas B-lineage lymphoma-b (Cbl-b) deficiency are also involved (Figure 1). Recent study has also reported that truncating mutation of $\beta$-2-microglobulin (B2M) is responsible for antigen presentation, resulting in resistance to anti-CTLA-4 and anti-PD-1 tumor therapy ${ }^{26}$.

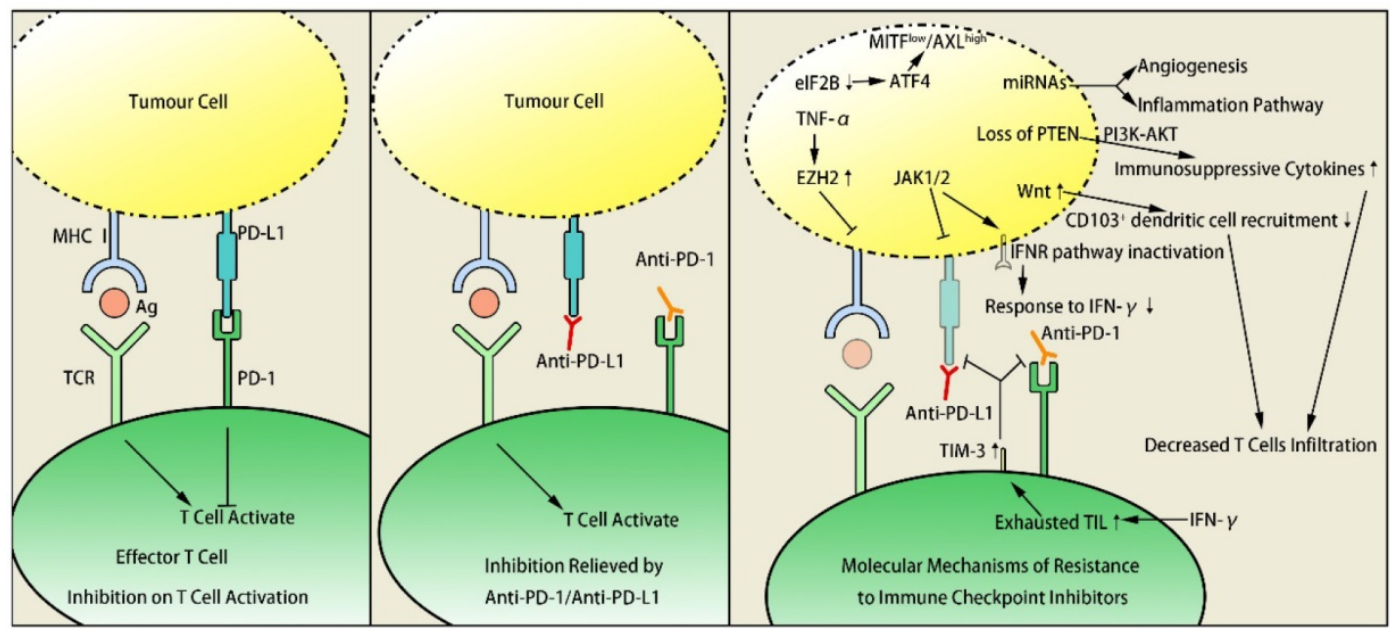

Figure 1. Molecular mechanisms of tumour resistance to immune checkpoint therapy 


\section{MITF suppression}

MITF is an oncogene of melanoma, which negatively correlates with tumor invasion ability. Low expression of MITF increases melanoma invasion, making tumor cell possess characteristics of tumor stem cells ${ }^{27}$, thus promotes the transformation of melanoma into a more invasive phenotype. This transformation contributes to tumor metastasis and immunotherapy resistance. Inhibition of translation initiation factor eIF2B leads ATF4 to activate AXL and suppress MITF, which increases MITFlow / AXLhigh drug-resistant phenotype, causing resistance in adoptive T-cell and anti-PD-1 immunotherapy ${ }^{28}$.

\section{Ezh2 upregulation}

Histone methyltransferase Ezh2 controls resistance to $\mathrm{T}$ cell immunotherapy as a molecular switch in melanoma ${ }^{29}$. $\mathrm{T}$ cell infiltration was positively associated with Ezh2-PRC2 complex activity in human skin melanoma cells according to research summary of Cancer Genome Atlas database. After treatment of anti-CTLA-4 mAbs or IL-2 in mice, intratumoral TNF- $\alpha$ and $\mathrm{T}$ cells accumulation promoted the Ezh2 expression, which resulted in tumor immunogenicity loss, antigen expression silence and immunotherapy resistance. It was concluded from this experiment that Ezh2 inactivation reversed the resistance, increased curative effect of anti-CTLA-4 mAbs or IL-2 and inhibited melanoma growth significantly.

\section{microRNA deregulation}

miRNAs control tumor cell signal conduction by regulation of target genes, functioning similar to oncogenes or anti-oncogenes. miRNAs not only influence tumorigenesis and development but also play a part in resistance to targeted or immune therapy ${ }^{30}$. Fattore $\mathrm{L}$ and his coworkers defined a group of miRNAs closely related to melanoma progressing and recognized main downstream pathways. miRNAs reveal acquired resistance to MAPKi as well as innate resistance to anti-PD-1 immunotherapy, which are both associated with alterations of angiogenesis and inflammatory pathways ${ }^{31,32}$. In lung cancer, miR-8 family is able to target the PD-L1, increasing the efficacy of $\mathrm{CD}^{+} \mathrm{T}$ cell and tumor immunosurveillance, meanwhile, the upregulation of the immune suppressive PD-L1/PD-1 axis could be caused by the downregulation of these miRNAs in melanoma $^{32}$. Expression of PD-L1 in lung cancer cells and melanoma cells could be unregulated by miR-8 family related miRNAs, causing immunotherapy resistance ${ }^{31}$.

\section{Loss of PTEN}

Research on melanoma in animal models confirmed that loss of PTEN decreased $\mathrm{T}$ cells infiltration and increased the expression of immunosuppressive cytokines at tumor sites. Loss of PTEN induces VEGF expression and subsequently leads to immune suppression, which is confirmed by clinical samples and B16 murine tumours that the blockade of VEGF results in increased trafficking of $\mathrm{T}$ cells $\mathrm{s}^{33}$. PTEN loss also inhibited autophagy, which decreased effect of T cell mediated tumor-killing and anti-PD-1 therapy ${ }^{34}$. Furthermore, human studies approved the strong relation between PTEN loss and pembrolizumab resistance ${ }^{35}$. Loss of PTEN promoted immune resistance through phosphatidylinositol 3-kinase (PI3K) - protein kinase B (AKT) pathway activation, which could be alternated by PI3K inhibitors. Therefore, combination of anti-PD-1 therapy and PI3K inhibitors applies to immunotherapy resistance from a new perspective ${ }^{34}$.

\section{TIM-3 upregulation}

Both PD-1 and TIM-3 are immunologic checkpoint receptors expressed on tumor infiltrating lymphocyte (TIL). TIM-3 is an inhibitory molecule which has close connection to immune tolerance and $\mathrm{T}$ cell exhaustion in tumours ${ }^{36}$, at the same time, its ligand Galectin-9 is secreted by epithelial cells in the thymus and mediates T cell apoptosis ${ }^{37}$. TIM-3 was significantly upregulated in TIL in tumor mouse models resistant to anti-PD- 1 therapy ${ }^{38}$. Clinical trials in adaptive resistant patients of anti-PD-1 therapy showed TIM-3 upregulation and disconnection of anti-PD-1 mAbs with T cells. TIM-3 was highly expressed in dysfunctional TIL in human HNSCC. Dampened AKT/S6 phosphorylation in PD-1+TIM- $3^{+}$ TIL led to weaken curative effect of anti-PD-1 treatment ${ }^{39}$. Adaptive resistance to anti-PD- $1 \mathrm{mAb}$ was overcome after addition of anti-TIM-3 mAb. This suggested TIM-3 and PI3K/AKT as promising targets of tumor immunotherapy.

\section{JAK 1/2 mutations}

JAK1/2 dysfunction induced by mutations leads to acquired resistance to anti-PD-1 therapy. In human melanoma cell lines lack of PD-L1 expression, JAK1/2 mutations were found in 2 out of 48 cell lines ${ }^{40}$. Patients with JAK1/2 loss-of-function alterations might present poor prognosis according to data of Cancer Genome Atlas database. In melanoma and dMMR colon cancer patients with anti-PD-1 resistance, high JAK1/2 mutation load were detected ${ }^{40}$. Zaretsky J.M also found similar function loss in metastatic melanoma patients, suggesting the association between acquired resistance to anti-PD-1 
therapy and JAK1/2 dysfunction ${ }^{41}$. The dysfunction of JAK1/2 led to PD-L1 expression absent or significantly down-regulated by IFN- $\gamma$ receptor pathway inactivation. Deficiency of immunotherapy target resulted in resistance to anti-PD-1/ PD-L1 mAbs. Combination of IFN $\gamma^{+} \mathrm{T}$ helper type 1 inducing cancer vaccines with PD-1 immune checkpoint blockade led to increase of PD-L1 expression ${ }^{42}$. The synergetic effect of combined therapy overcame immunotherapy resistance to some extent.

\section{Cbl-b deficiency}

Cbl-b is an E3 ubiquitin ligase that regulates T cell activation negatively. PD-L1 Ig not only suppresses $\mathrm{T}$ cell proliferation but also inhibits secretion of IFN- $\gamma$. Study on mice showed that Cbl-b WT mice were sensitive to PD-L1 Ig while Cbl-b/mice were not. In coculture of $\mathrm{Cbl-b}-/-\mathrm{CD} 8^{+} \mathrm{T}$ cells and WT CD8 ${ }^{+} \mathrm{T}$ cells, the existence of $\mathrm{Cbl-b} / \mathrm{CD}^{-\mathrm{C}}{ }^{+} \mathrm{T}$ cells diminished PD-L1 Ig mediated suppression of WT $\mathrm{CD}^{+} \mathrm{T}$ cells. WT mice had increased sensitivity to treatment and fewer liver metastases while Cbl-b/mice showed no curative effect. The experimental results suggested that the $\mathrm{Cbl}-\mathrm{b}^{-/}$- phenotype is one of the key factors of anti-PD-L1/PD-1 resistance or tumor progression ${ }^{43}$.

\section{B2M truncating mutation}

$\mathrm{B} 2 \mathrm{M}$ is associated with the heavy chain of major histocompatibility complex (MHC) I and the mutations of B2M may impact MHC I antigen presentation. Point mutations, deletions or loss of heterozygosity were detected in $29.4 \%$ in 17 progressing metastatic melanoma patients treated with ICBs. Also, the study suggested that B2M loss of heterozygosity enriched threefold in non-responders (30\%) compared to responders $(10 \%)$ along with poorer overall survival in independent cohorts of melanoma patients treated with anti-CTLA4 and anti-PD1 respectively. It is concluded that the occurrence of $\mathrm{B} 2 \mathrm{M}$ truncating mutation results in resistance to immunotherapy ${ }^{26}$.

\section{Wnt/ $\beta$-catenin signaling pathway activation}

The activity changing of Wnt signaling is involved in multiple human diseases, including cancer, bone defects, schizophrenia, and arthritis ${ }^{44}$. Wnt1gene, a secreted cysteine-rich protein with the potential to act as a signaling molecule, is confirmed to induce breast tumor in mouse models ${ }^{45}$. Spranger revealed the correlation between activation of WNT/ $\beta$-catenin signaling pathway and absence of T-cell gene expression signature in human metastatic melanoma samples ${ }^{46}$. By mediating immune exclusion in melanoma caused by defective recruitment of CD1031 dendritic cells, the activation of Wnt $/ \beta$-catenin signaling pathway resulted in resistance of immune-based therapies including anti-CTLA-4 and anti-PD-L1 mAbs ${ }^{46}$.

\section{Other mechanisms}

Study on metastatic melanoma patient collected samples from 13 anti-PD-1 resistant patients and 3 patients resistant to combination of anti-PD-1 and ipilimumab therapy. In most resistant patients, density of VISTA ${ }^{+}(12 / 18)$, PD-L1 expression (11/18) and intratumoral expression of $\mathrm{FOXP3}^{+}$were detected. In a small part of patients, PTEN loss (5/18), downregulation of HLA-A (4/18) and HLA-DPB1 $(3 / 18)$ were found, which suggested the close relations between above indexes and acquired resistance to anti-PD-1 treatment ${ }^{47}$. Vivo imaging could reveal the real-time activity of anti-PD-1 mAbs at subcellular resolution in mice. Tumor-associated macrophages engulfed anti-PD-1 mAbs swiftly and the phagocytosis depended both on mAbs' Fc domain glycan and $\mathrm{F}_{\mathrm{C}}$ receptors expressed by host myeloid cells. Before applying anti-PD-1 mAbs, blockade of $\mathrm{Fcy}$ receptors prolonged the combining of $\mathrm{mAbs}$ and tumor-infiltrating $\mathrm{CD}^{+} \mathrm{T}$ cells and enhanced tumor regression $^{48}$. Loss of IFN- $\gamma$ genes were found in ipilimumab resistant patients while knockdown of IFN- $\gamma$ receptor 1 could impair the curative effect of anti-CTLA-4 therapy, indicating defect of IFN- $\gamma$ pathways as a mechanism of anti-CTLA-4 therapy resistance ${ }^{49}$.

\section{Countermeasures of immunotherapy resistance}

There are many strategies to overcome the resistance to immunotherapy, such as combination of chemotherapy, radiotherapy and tumor vaccine, etc. Agonists and inhibitors of specific receptors and molecules can also be applied to optimize immune therapy and overcome resistance.

\section{PRRs agonist}

Pattern recognition receptors (PRRs), a kind of pathogen associated molecular patterns recognition molecule on surface of innate immune cells, have effects of regulatory, phagocytosis, complement activation, inflammatory signal transduction, and inducing apoptosis. PRRs act as costimulatory molecules of macrophages and dendritic cells. Natural endo/ exogenous or synthetic PRRs agonists activate phagocytosis and antigen presentation of macrophages and myeloid cells in the tumor microenvironment. Preclinical models proved that PRRs agonists could overcome the resistance to anti-CTLA-4, anti-PD-1 and anti-PD-L1 targeting T-cell immune checkpoints ${ }^{50}$. 


\section{ARNAX}

ARNAX, a tumor vaccine, can induce anti-tumor CTLs without systemic cytokine/interferon production by increasing the efficacy of anti-PD-L1 as Toll-like receptor 3 agonist. It can also improve immunity of $\mathrm{T}$ helper type 1 in tumor microenvironment and enhance recruitment of dendritic cells, $\mathrm{T}$ cells and natural killer cells. Combination of ARNAX and anti-PD-L1 activates tumor-specific CTL then enhances CTL infiltration, which would overcome resistance to anti-PD-L1. Human research showed $\mathrm{ARNAX}^{+}$antigen induced and increased proliferation of antigen-specific CTL in peripheral blood mononuclear cells, indicating the potential of ARNAX to synergize anti-PD-1/PD-L151.

\section{CpG oligonucleotide}

CpG oligonucleotide function as a stimulator in the presence of anti-PD-1, inducing IFN, T-cell-tropic chemokines, and DC maturation thereby generating systemic $\mathrm{T}$ cell response to infiltrate tumor ${ }^{52}$. AntiPD-1 treatment induces CpG-mediated tumor-specific $\mathrm{CD}^{+} \mathrm{T}$ cells to differentiate into $\mathrm{CD} 127$ highKLRG1 low long-lived memory precursors preferentially. Intratumoral $\mathrm{CpG}$ oligonucleotide administration improves the quantity and quality of tumor-specific $\mathrm{CD}^{+} \mathrm{T}$ cells, increasing sensitivity of anti-PD-152.

\section{Oncolytic peptide LTX-315}

The oncolytic peptide LTX-315 could promote the release of danger signals (DAMPs) such as ATP, Cytochrome $\mathrm{C}$ and HMGB1 and then cause plasma membrane disruption, mitochondria perturbation as well as cell death, acting as an immunogenic cytotoxic compound ${ }^{53}$. LTX-315 improves antitumor immunity by decreasing local immune suppressive $T$ regulatory cells and myeloid-derived suppressor cells, leading to increase in CTLA-4 and decrease in PD-1 expression. The study of Yamazaki proved that intratumoral rejection of LTX-315 caused tumor regression or disappearance, offering the theoretical support to the combination of ipilimumab and LTX-31554.

\section{IDO inhibitors}

Indoleamine 2,3-dioxygenase (IDO) is the rate-limiting enzyme of tryptophan catabolism, which is highly expressed in immune tolerant tumor tissues. In melanoma cells in IDO knockout mice treated with anti-CTLA-4 mAbs, tumor proliferation was inhibited and overall survival increased compared with wildtype mice, which was also observed in anti-PD-1/ PD-L1 and glucocorticoid-induced tumor necrosis factor receptor therapy ${ }^{55}$. Combination of anti-CTLA$4 \mathrm{mAbs}$ and IDO inhibitors reduced the inhibition of
IDO on T cells and enhanced tumor-specific effector T cells infiltration. Hence, combination of IDO inhibitors is able to increase therapeutic effect of $\mathrm{ICBs}^{55}$. The synergistic effect has been approved by multiple clinical trials $56,57,58$.

\section{Combination of radiotherapy}

During anti-PD-1 treatment in Kras-mutated p53-deficient lung cancer murine, PD-L1 expression on resistant and sensitive tumor cell showed no obvious difference. MHC I/II molecules on resistant tumor cells was downregulated significantly, along with TIL and IFN- $\gamma$ decrease in microenvironment compared to sensitive tumor cells. However, local radiotherapy promoted IFN- $\beta$ secretion and upregulated expression of MHC I molecules on the surface of resistant cells. Results proved that adjuvant radiotherapy helps to get over anti-PD-1 resistance, and then enhances efficacy of anti PD-1 immunotherapy ${ }^{59}$.

\section{anti-TIM3 Ab TSR-022}

Upregulation of second immune checkpoint TIM-3 has been discovered in anti-PD-1 Ab-resistant tumors $^{39}$. The Phase I clinical trial of anti-TIM3 Ab TSR-022 is ongoing (ID\# NCT02817633), as a monotherapy and in combination with an anti-PD-1 antibody, in patients with advanced solid tumors who have limited available treatment options. Further experiments are required for anti-TIM3 $\mathrm{Ab}$ to clarify its unique value.

\section{anti-LAG-3 Ab}

Lymphocyte activation gene 3 (LAG-3) is a gene functioning as an inhibitory receptor on T cells, which increases the effect of regulatory $\mathrm{T}$ cells and shows relationship with $\mathrm{T}$ cell exhaustion ${ }^{60}$. Combining anti-LAG-3 $\mathrm{Ab}$ and anti-PD-1 Ab synergistically enhances T cell activity, and phase I/II clinical trial about the combination treatment is ongoing (ID\#NCT01968109).

\section{Other countermeasures}

In addition to above strategies, gene detection ${ }^{21,22}$, blocking PI3K-AKT, blockade of $\mathrm{Fcy}$ receptors ${ }^{47}$ and modulation of the gut microbiome ${ }^{61}$ are also feasible. In patients with PD-1 resistant melanoma, upregulation of a few immune related genes results in immunosuppression ${ }^{32}$, angiogene$\mathrm{sis}^{34}$, monocyte and macrophage chemotaxis ${ }^{49}$, extracellular matrix remodeling 61 and epithelial mesenchymal transition ${ }^{61,62}$. Therefore, combined blockade of these genes and PD-1 may administer to overcome anti-PD-1 resistance, providing better effects of antitumor immunotherapy ${ }^{63}$ (Table 1) (Figure 2). 


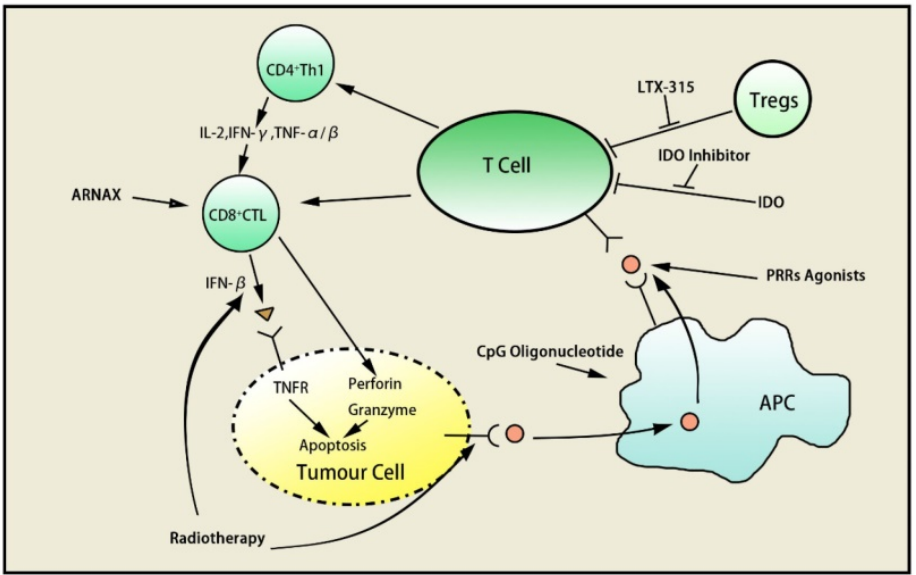

Figure 2. Countermeasures of immunotherapy resistance

Table 1. Strategies to overcome resistance to immune checkpoints blockade in cancer

\begin{tabular}{llll}
\hline mechanism & target blocking & T cell synergism & antigen presentation majorization \\
\hline strategy & TSR-022 & ARNAX & PRRs agonist \\
& blocking PI3K-AKT & CpG oligonucleotide & radiotherapy \\
& blocking FcyR & IDO inhibitors & \\
gene detection & LTX-315 & \\
\hline
\end{tabular}

\section{Conclusion and perspectives}

Immunotherapy is a new choice for cancer patients and has an unparalleled advantage compared to traditional treatment. Overcoming resistance to immunotherapy helps patients to get more benefit from this novel treatment thereby improving survival and life quality ultimately. Choosing valid biomarkers, whole-exome sequencing, analysis of gene expression, and seeing the whole forest of immunotherapy contributes to responders identification, resistance overcoming and resistant patients cure, which worth further exploration in the future.

\section{Abbreviations}

ICBs: immune checkpoint blockers; mAb: monoclonal antibody; CAR-T: chimeric antigen receptor T-cell immunotherapy; NSCLC: non-small cell lung cancer; HNSCC: head and neck squamous cell carcinoma; MITF: microphthalmia-associated transcription factor; Ezh2: enhancer of zeste homolog 2; TIM-3: T cell immunoglobulin mucin-3; Cbl-b: Casitas Blineage lymphoma-b; B2M: $\beta$-2-microglobulin; PI3K: phosphatidylinositol 3-kinase; AKT: protein kinase B; TIL: tumor infiltrating lymphocyte; PRRs: pattern recognition receptors; IDO: indoleamine 2,3-dioxygenase.

\section{Acknowledgements}

Thanks Seyed Kariminia for revision of English and polishing.

\section{Funding}

This review is funded by the National Natural Science Foundation of China (Grant NO. 81301912), Beijing Municipal Health System High-level Health Person Foundation Project (Grant NO. 2014-3-005), the Beijing Municipal Science and Technology Commission Foundation (Capital Features, Z16110000 0516083, to Qin Li) and Natural Science Foundation of Capital Medical University (to Qin Li).

\section{Author Contributions}

Qin Li contributed to the design of the review and revised the article, had full access to all of the contents included in this study and took responsibility for the integrity of the data and the accuracy of the data analysis. Li Li provided the guide of immune knowledge, Xiaoyue Jiang wrote the main manuscript text and supplied the figures. All authors agreed to be accountable for the content of this paper.

\section{Competing Interests}

The authors have declared that no competing interest exists.

\section{References}

1. Yang Y. Cancer immunotherapy: harnessing the immune system to battle cancer. J Clin Invest.2015; 125(9):3335-7.

2. Ascierto PA, Vanella V, Grimaldi AM, et al. Complete response to nivolumab monotherapy in a treatment-naive, BRAF wild-type patient with advanced mucosal melanoma and elevated lactate dehydrogenase: a case report from a phase III trial. Cancer Immunol Immun. 2016; 65(11):1395. 
3. Weber J, Mandala M, Del MV, et al. Adjuvant Nivolumab versus Ipilimumab in resected stage III or IV Melanoma. N Engl J Med. 2017; 377(19):1824-35.

4. Hodi FS, O'Day SJ, Mcdermott DF, et al. Improved survival with ipilimumab in patients with metastatic melanoma. N Engl J Med, 2010; 363(8):711-23.

5. Borghaei H, Pazares L, Horn L, et al. Nivolumab versus Docetaxel in advanced nonsquamous Non-Small-Cell Lung Cancer. N Engl J Med. 2015; 373(17):1627-39.

6. Shaverdian $\mathrm{N}$, Lisberg AE, Bornazyan $\mathrm{K}$, et al. Previous radiotherapy and the clinical activity and toxicity of pembrolizumab in the treatment of non-small-cell lung cancer: A secondary analysis of the KEYNOTE-001 phase 1 trial. Lancet Oncol. 2017; 18(7):895.

7. Ferris RL, Blumenschein G, Fayette J, et al. Nivolumab for recurrent squamous-cell carcinoma of the head and neck. N Engl J Med. 2016; 375(19): 1856

8. Melero I, Crocenzi TS, Welling TH, et al. Phase I/II safety and antitumor activity of nivolumab in patients with advanced hepatocellular carcinoma (HCC): CA209-040. J Clin Oncol. 2015; 33(Suppl 18): LBA101.

9. Overman MJ, Mcdermott R, Leach JL, et al. Nivolumab in patients with metastatic DNA mismatch repair-deficient or microsatellite instability-high colorectal cancer (CheckMate 142): an open-label, multicentre, phase 2 study. Lancet Oncol. 2017; 18(9):1182.

10. Sharma P, Callahan MK, Bono P, et al. Nivolumab monotherapy in recurrent metastatic urothelial carcinoma (CheckMate 032): a multicentre, open-label, phase $1 / 2$ trial. Lancet Oncol. 2016; 17(11):1590-98.

11. Motzer RJ, Rini BI, Mcdermott DF, et al. Nivolumab for metastatic renal cell carcinoma: Results of a randomized phase II Trial. J Clin Oncol. 2015; 33(13):1430.

12. Younes A, Santoro A, Shipp M, et al. Nivolumab for classical Hodgkin's lymphoma after failure of both autologous stem-cell transplantation and brentuximab vedotin: a multicentre, multicohort, single-arm phase 2 trial. Lancet Oncol. 2016; 17(9):1283-94.

13. Fuchs CS, Doi T, Jang RW, et al. Safety and efficacy of Pembrolizumab monotherapy in patients with previously treated advanced gastric and gastroesophageal junction cancer: Phase 2 clinical KEYNOTE-059 Trial. JAMA Oncol. 2018; 4(5).

14. Qin $\mathrm{ZM}, \mathrm{Fu} \mathrm{CH}$, Zhang $\mathrm{F}$, et al. Efficacy and safety of antibodies targeting PD-1 / PD-L1 inhibitors versus chemotherapy in the treatment of advanced non-small cell lung cancer: A meta-analysis. J Mod Oncol. 2017; 25(22): 3613-19.

15. Weber J, Thompson JA, Hamid O, et al: A randomized, double-blind, placebo-controlled, phase II study comparing the tolerability and efficacy of ipilimumab administered with or without prophylactic budesonide in patients with unresectable stage III or IV melanoma. Clin Cancer Res. 2009; 15:5591-98

16. O'Day SJ, Maio M, Chiarion-Sileni V, et al: Efficacy and safety of ipilimumab monotherapy in patients with previously treated, advanced melanoma: A multicenter, single-arm, phase II study. Ann Oncol 2010; 21:1712-17.

17. Wolchok JD, Neyns B, Linette G, et al: Ipilimumab monotherapy in patients with previously treated, advancedmelanoma: A randomized, double-blind, multicenter, phase 2, dose-ranging study. Lancet Oncol. 2010; 11:155-64.

18. Schadendorf D, Hodi FS, Robert C, et al. Pooled analysis of long-term survival data from phase II and phase III trials of Ipilimumab in unresectable or metastatic melanoma. J Clin Oncol. 2015; 33(17):1889-94.

19. Ribas A, Hamid O, Daud A, et al. Association of Pembrolizumab with tumor response and survival among patients with advanced melanoma. JAMA. 2016; 315(15):1600.

20. Jansen T, Bmch-Gerharz D, Reifenberger J, Schuhe KW, Metastatic malignant melanoma: Sueeessfull treatment with ipilimumab. Hautarzt. 2013; 64(4) : 228-31.

21. Snyder A, Makarov V, Hellmann M, et al. Genetics and immunology: reinvigorated. OncoImmunology. 2015; 4(10): e1029705.

22. Milano G. Resistance to immunotherapy: Clouds in a bright sky. Invest. New Drugs. 2017; 17:1-6.

23. Wilmott JS, Rizos H, Scolyer RA, et al. The "tricky business" of identifying mechanisms of resistance to anti-PD-1. Clin. Cancer Res. 2017; 23(12).

24. Andrews MC, Wargo JA. Immunotherapy resistance: The answers lie ahead - not in front - of us. J ImmunoTher Cancer. 2017; 5(1):10.

25. Ascierto ML, Makohonmoore A, Lipson EJ, et al. Transcriptional mechanisms of resistance to anti-PD-1 therapy. Clin. Cancer Res. 2017; 23(12):3168.

26. Sade-Feldman $\mathrm{M}$, Jiao $\mathrm{YJ}$, Chen $\mathrm{JH}$, et al. Resistance to checkpoint blockade therapy through inactivation of antigen presentation: Nat. Commun. 2017; 8(1):1136.
27. Fang MJ, Yan K, Guo J, et al. Biological and clinical research progress of MITF in melanoma. Tumor. 2013; 33(12).

28. Falletta P, Sanchezdelcampo L, Chauhan J, et al. Translation reprogramming is an evolutionarily conserved driver of phenotypic plasticity and therapeutic resistance in melanoma. Genes Dev. 2017; 31(1):18.

29. Zingg D, Arenasramirez N, Sahin D, et al. The Histone Methyltransferase Ezh2 Controls Mechanisms of Adaptive Resistance to Tumor Immunotherapy. Cell Rep. 2017; 20(4):854.

30. Voorhoeve PM,le Sage C,Schrier M, et al. A genetic screen implicates miRNA-372and miRNA-373as oncogenes in testicular germ cell tumors. Cell. 2006;124(6):1169-81

31. Fattore L, Sacconi A, Mancini R, et al. MicroRNA-driven deregulation of cytokine expression helps development of drug resistance in metastatic melanoma. Cytokine Growth Factor Rev. 2017; 36: 39-48.

32. Chen L, Gibbons DL, Goswami S, et al. Metastasis is regulated via microRNA-200/ZEB1 axis control of tumour cell PD-L1 expression and intratumoral immunosuppression. Nat. Commun. 2014; 5: 5241.

33. Shrimali RK, Yu Z, Theoret MR, et al. Antiangiogenic agents can increase lymphocyte infiltration into tumor and enhance the effectiveness of adoptive immunotherapy of cancer. Cancer Res. 2010; 70(15):6171.

34. Peng W, Chen JQ, Liu C, et al. Loss of PTEN Promotes Resistance to T Cell-Mediated Immunotherapy. Cancer Discov. 2016; 6(2):202.

35. George S, Miao D, Demetri GD, et al. Loss of PTEN Is Associated with Resistance to Anti-PD-1 Checkpoint Blockade Therapy in Metastatic Uterine Leiomyosarcoma. Immunity. 2017; 46(2):197.

36. Huang $\mathrm{YH}, \mathrm{Zhu} \mathrm{C}$, Kondo $\mathrm{Y}$, et al. CEACAM1 regulates TIM-3-mediated tolerance and exhaustion. Nature. 2015; 517(7534):386-90.

37. Hernandez JD, Baum LG. Ah, sweet mystery of death! Galectins and control of cell fate. Glycobiology. 2002; 12(10):127R.

38. Shohei K, Akbay EA, Li YY, et al. Adaptive resistance to therapeutic PD-1 blockade is associated with upregulation of alternative immune checkpoints. Nat Commun. 2016; 7:10501.

39. Shayan G, Srivastava $\mathrm{R}, \mathrm{Li}$ J, et al. Adaptive resistance to anti-PD1 therapy by TIM-3 upregulation is mediated by the PI3K-Akt pathway in head and neck cancer. Oncoimmunology. 2016; 6(1):e1261779.

40. Shin DS, Zaretsky JM, Escuin-Ordinas H, et al. Primary resistance to PD-1 blockade mediated by JAK1/2 mutations. Cancer Discov. 2017; 7(2):188.

41. Zaretsky JM, Garcia-Diaz A, Shin DS, et al. Mutations associated with acquired resistance to PD-1 blockade in melanoma. N Engl J Med. 2016; 375(9):819.

42. Kim YJ. Subverting the adaptive immune resistance mechanism to improve clinical responses to immune checkpoint blockade therapy. Oncoimmunology. 2015; 3(12):e954868.

43. Mai F, Anstadt EJ, Clark RB. Cbl-b Deficiency Mediates Resistance to Programmed Death-Ligand 1/Programmed Death-1 Regulation. Front Immuno. 2017; 8(6766).

44. Clevers $\mathrm{H}$. Wnt/beta-catenin signaling in development and disease. Cell. 2006, 127(3):469-80.

45. Nusse R, Varmus HE. Many tumors induced by the mouse mammary tumor virus contain a provirus integrated in the same region of the host genome. Cell. 1982; 31(1):99-109.

46. Spranger S, Bao R, Gajewski TF. Melanoma-intrinsic $\beta$-catenin signalling prevents anti-tumour immunity. Nature. 2015; 523(7559):231-35.

47. Kakavand H, Jackett LA, Menzies AM, et al. Negative immune checkpoint regulation by VISTA: a mechanism of acquired resistance to anti-PD-1 therapy in metastatic melanoma patients. Modern Pathol. 2017; 30(12):1666-76.

48. Arlauckas SP, Garris CS, Kohler RH, et al. In vivo imaging reveals a tumor-associated macrophage-mediated resistance pathway in anti-PD-1 therapy. Sci. Transl. Med. 2017; 9(389): eaal3604.

49. Gao J, Shi LZ, Zhao H, et al. Loss of IFN- $\gamma$ pathway genes in tumor cells as a mechanism of resistance to anti-CTLA-4 therapy. Cell. 2016; 167(2):397-404

50. Shekarian T, Valsesia-Wittmann S, Brody J, et al. Pattern recognition receptors: immune targets to enhance cancer immunotherapy. Ann Oncol. 2017; 28(8): 1756-1766.

51. Takeda Y, Kataoka K, Yamagishi J, et al. A TLR3-specific adjuvant relieves innate resistance to PD-L1 blockade without cytokine toxicity in tumor vaccine immunotherapy. Cell Rep. 2017; 19(9):1874.

52. Wang S, Campos J, Gallotta M, et al. Intratumoral injection of a CpG oligonucleotide reverts resistance to PD-1 blockade by expanding multifunctional CD8+ T cells. PNAS. 2016; 113(46): E7240.

53. Liv-Marie E, Yang N, Øystein R, et al. The oncolytic peptide LTX-315 induces cell death and DAMP release by mitochondria distortion in human melanoma cells. Oncotarget. 2015; 6(33):34910-23. 
54. Yamazaki T, Pitt JM, Vétizou M, et al. The oncolytic peptide LTX-315 overcomes resistance of cancers to immunotherapy with CTLA4 checkpoint blockade. Cell Death Differ. 2016; 23(6):1004.

55. Holmgaard RB, Zamarin D, Munn DH, et al. Indoleamine 2,3-dioxygenase is a critical resistance mechanism in antitumor $\mathrm{T}$ cell immunotherapy targeting CTLA-4. J ImmunoTher Cancer. 2013; 1(S1):1389-402.

56. Prendergast GC, Smith C, Thomas S, et al. Indoleamine 2,3-dioxygenase pathways of pathgenic inflammation and immune escape in cancer. Cancer Immunol Immun. 2014; 63(7):721.

57. Bjoern J, Iversen TZ, Nitschke NJ, et al. Safety, immune and clinical responses in metastatic melanoma patients vaccinated with a long peptide derived from indoleamine 2,3-dioxygenase in combination with ipilimumab. Cytotherapy. 2016; 18(8):1043-55.

58. Yu J, Wang Y, Yan F, et al. Noncanonical NF-kB activation mediates STAT3-stimulated IDO upregulation in myeloid-derived suppressor cells in breast cancer. J Immunol. 2014; 193(5):2574.

59. Wang X, Schoenhals JE, Li A, et al. Suppression of type I IFN signaling in tumors mediates resistance to anti-PD-1 treatment that can be overcome by radiotherapy. Cancer Res. 2017; 77(4):839.

60. Wherry EJ. T cell exhaustion. Nat Immunol. 2011; 12(6):492.

61. Pitt JM, Vétizou $M$, Daillère $R$, et al. Resistance mechanisms to immune-checkpoint blockade in cancer: Tumor-intrinsic and -extrinsic factors. Immunity. 2016; 44(6):1255.

62. Hugo W, Zaretsky JM, Sun L, et al. Genomic and transcriptomic features of response to anti-PD-1 therapy in metastatic melanoma. Cell. 2016; 165(1): 35-44.

63. Bu X, Mahoney KM, Freeman GJ. Learning from PD-1 resistance: New combination strategies. Trends Mol Med. 2016; 22(6):448. 